\title{
Solid-state binding, recombination, and Auger energy shifts of rare-earth metals
}

\author{
M. Richter, T. Prescher, M. Meyer, E. v. Raven, B. Sonntag, and H. E. Wetzel \\ II. Institut für Experimentalphysik, Universität Hamburg, D-2000 Hamburg 50, West Germany \\ S. Aksela \\ Department of Physics, University of Oulu, SF-90570 Oulu 57, Finland
}

(Received 19 January 1988)

\begin{abstract}
The free-atom binding energies up to $4 d$ core levels have been determined from monochromatic synchrotron-radiation-excited photoelectron spectra for the elements $\mathrm{La}, \mathrm{Ce}, \mathrm{Nd}, \mathrm{Sm}, \mathrm{Eu}$, and $\mathrm{Gd}$. They are compared with the corresponding solid-state values, and the atom-to-metal binding energy shifts are discussed in comparison with the theoretical calculations available. Atomic and solidstate $4 d$-based Auger and direct recombination (autoionization) spectra of the elements $\mathrm{La}, \mathrm{Ce}, \mathrm{Pr}$, $\mathrm{Nd}, \mathrm{Sm}, \mathrm{Eu}, \mathrm{Gd}, \mathrm{Tb}, \mathrm{Dy}$, and $\mathrm{Tm}$ have also been studied and are compared in detail. The spectra show clearly very different solid-state shifts for the $4 d$-based Auger and $4 d \rightarrow(4 f, \epsilon f)$ autoionization peaks indicating a very useful method for distinguishing these two processes in electron-excited spectra. The intensities of the recombination peaks in the spectra of $\mathrm{La}, \mathrm{Ce}, \mathrm{Sm}, \mathrm{Eu}$, and $\mathrm{Gd}$ were also measured, using the white-light synchrotron radiation obtained in zeroth order from a toroidal-grating monochromator, and were found to be increased in intensity. The autoionization and Auger energy shifts are discussed in terms of theoretical models.
\end{abstract}

\section{INTRODUCTION}

The binding-energy shifts of $4 f$ elements have attracted considerable theoretical and experimental interest. One reason for this is the valence change found for some lanthanides upon condensation of free atoms to solids and, on the other hand, between bulk and surface states of the same metallic elements. Furthermore, the valence changes have been found to take place between different compounds of given lanthanide elements. In this study we will deal with experimental free-atom-to-metal binding, Auger, and direct recombination energy shifts of $\mathrm{La}$ and several $4 f$ elements. Extensive comparisons based on direct experimental results have not been possible earlier due to the lack of much needed free-atom spectra. The measurement of free-atom spectra has been hampered by both the considerable difficulties arising from the high temperatures needed to produce the necessary vapor pressures and the high reactivities of some lanthanides as liquids and vapors.

Electron-beam-excited electron emission spectra of lanthanides have, apart from some $3 d$ metals, proved to be exceptional because the spectra show in addition to the conventional Auger transitions strong direct recombination peaks from core excitations. The appearance of direct recombination or autoionization lines was first suggested by Dufour and Bonnelle ${ }^{1,2}$ and later confirmed by several authors. ${ }^{3-7}$ The $4 d-4 f$ and $4 d-5 p$ direct recombination spectra appear on the high-kinetic-energy side of the very broad $4 d$-based Auger peaks and therefore their unambiguous identification is often difficult.

The direct recombination lines originate from $4 d \rightarrow(4 f, \epsilon f)$ giant-resonance excitations observed both in the $x$-ray-absorption, ${ }^{8,9}$ photoelectron, ${ }^{10,11}$ and electron-energy-loss spectra ${ }^{12}$ of metallic lanthanides, as well as in free-atom spectra. ${ }^{13-18} 4 d \rightarrow(4 f, \epsilon f)$ giant resonances of the rare-earth metals are many-electron phenomena which can be thought of as arising from large overlap between the occupied $4 d$ and unoccupied $4 f$ wave function. The overlap of the wave functions results in a strong coupling of the $4 d$ core-excited $\left[4 d^{10} 4 f^{n} \rightarrow 4 d^{9}(4 f, \epsilon f)^{n+1}\right]$ states with the $4 f$ ionization $\left(4 f^{n} \rightarrow 4 f^{n-1}, \epsilon l\right)$ continua. These interferences manifest themselves as asymmetric resonance profiles in the total and partial cross sections. Other important decay channels of these giant excitation resonances are the recombination processes leading to the emission of a $5 p$ or $5 s$ electron. High-energy electron beams which correspond to a white light source can also produce $4 d \rightarrow(4 f, \epsilon f)$ resonance excitations. The large cross sections at the giant resonances result in intensities from the autoionization decay channels which are comparable to normal Auger processes created mainly at higher photon energies via $4 d$ ionization. An excellent compilation of recent review articles on giant resonances has been edited by Connerade et al. ${ }^{19}$

The white light of the synchrotron radiation, reflected undispersed (zeroth order) from the grating, produces, analogously with high-energy electron beams, both ionizations and resonance excitations. However, the intensity profile of the synchrotron radiation as a function of energy is manifested in the intensity ratio between autoionization and normal Auger lines being different from that obtained by electron-impact excitation.

In this study we wish to compare electron-excited solid-state rare-earth-metal ejected-electron spectra with electron- and/or white-synchrotron-radiation-excited atomic ejected-electron spectra with special emphasis on the metal-to-atom kinetic-energy shifts in corresponding Auger and autoionization lines. 
Kinetic-energy shifts between the solid and vapor phases of the $4 d$-based Auger and autoionization spectra of rare-earth metals are interesting for two main reasons. First, the contributions to the metal-to-atom kineticenergy shifts resulting from the extra-atomic relaxation energies in the metallic state should be very different for the Auger lines and autoionization lines. The Auger process creates a double-hole final state which is more strongly screened by the surrounding electronic charge than in the single-ionized states, in the initial state, or in the final state of the autoionization process. Different shifts of Auger and autoionization lines were pointed out by Netzer et $a l .{ }^{3}$ in studying the spectra of clean and oxidized solid Sm. Second, a number of the rare-earth atoms ( $\mathrm{Pr}$ to $\mathrm{Sm}$ and $\mathrm{Tb}$ to $\mathrm{Tm}$ ) undergo changes in their electron configurations upon condensation to solid metal which should also influence the metal-to-atom Auger and recombination line energy shifts.

Theoretical studies of atom-to-metal binding-energy shifts for La and $4 f$ elements have been performed by Herbst $^{20}$ and Johansson et al., ${ }^{21,22}$ but direct comparisons with experimental data have not been possible until now. Recently, Meyer et al. ${ }^{17}$ have presented the $4 d$ based ejected-electron spectra of these elements and a comprehensive study of the spectra of solid lanthanides has been presented by Riviere et al. ${ }^{23}$ The discussion in the present paper is based, for solids, on these experimental results, whereas for the vapor-phase binding energies obtained from photoelectron spectra ${ }^{24}$ are used.

The theoretical calculations of Herbst ${ }^{20}$ predict clearly different binding-energy shifts for various $n l$ subshells when the rare-earth metals have differing electron configurations as free atoms and as a solid. On the other hand, the semiempirical thermochemical model ${ }^{21}$ used very successfully to predict atom-to-metal shift for many elements can give only one common value which is supposed to be valid for different $n l$ binding energies. Therefore it was interesting to experimentally test the dependence of the solid-state shifts for different $n l$ subshells. The atom-to-metal $L$-level binding-energy shifts determined from absorption data ${ }^{25}$ were in agreement with the predictions of both models.

Different models to predict metal-to-atom Auger energy shifts have been used ${ }^{26-34}$ successfully for lighter elements. Among those most commonly used are the procedures based on excited-atom models, ${ }^{26,27}$ the selfconsistent-field ( $\triangle \mathrm{SCF})$ density-functional formalism, ${ }^{28-30}$ and thermochemical models applied also to Auger shifts. ${ }^{22,31,32}$ The basic problem in the present case of lanthanides is the complexity of the spectra and calculations due to the partially filled $4 f$ subshell.

\section{EXPERIMENT}

Most of the vapor Auger spectra to be discussed were obtained using electron-beam excitation $(1.5 \mathrm{keV}, 1 \mu \mathrm{A})$. The vapor-phase spectra were measured in HASYLAB at DESY, Hamburg, using the cylindrical-mirror analyzer at the energy resolution of $0.78 \%$; the electron-beamheated high-temperature oven ${ }^{35}$ was applied in these measurements.
In addition, the vapor-phase spectra of $\mathrm{Sm}, \mathrm{Eu}, \mathrm{Gd}$, and Tm were measured at the University of Oulu using a cylindrical-mirror analyzer and an inductively heated metal-vapor system; the energy resolution in these measurements was $0.05 \%$ and the typical primary electronbeam parameters were $3 \mathrm{keV}$ and $1 \mathrm{~mA}$. The agreement between the two vapor-phase measurements is very good.

The vapor-phase binding energies of the atomic outercore levels up to $4 d$ were obtained $^{24}$ for $\mathrm{La}, \mathrm{Ce}, \mathrm{Nd}, \mathrm{Sm}$, $\mathrm{Eu}$, and $\mathrm{Gd}$ from photoelectron spectra excited by synchrotron radiation from the BESSY storage ring in Berlin, monochromatized by a toroidal-grating monochromator (TGM) $(\Delta E / E \simeq 350)$. Using the white-light synchrotron radiation obtained in zeroth order from the TGM, Auger spectra have been measured in addition to the electron excitation spectra.

The solid-state spectra have been taken from the comprehensive study of Riviere et al. ${ }^{23}$ In the energy calibration of the spectra, vacuum and Fermi levels have

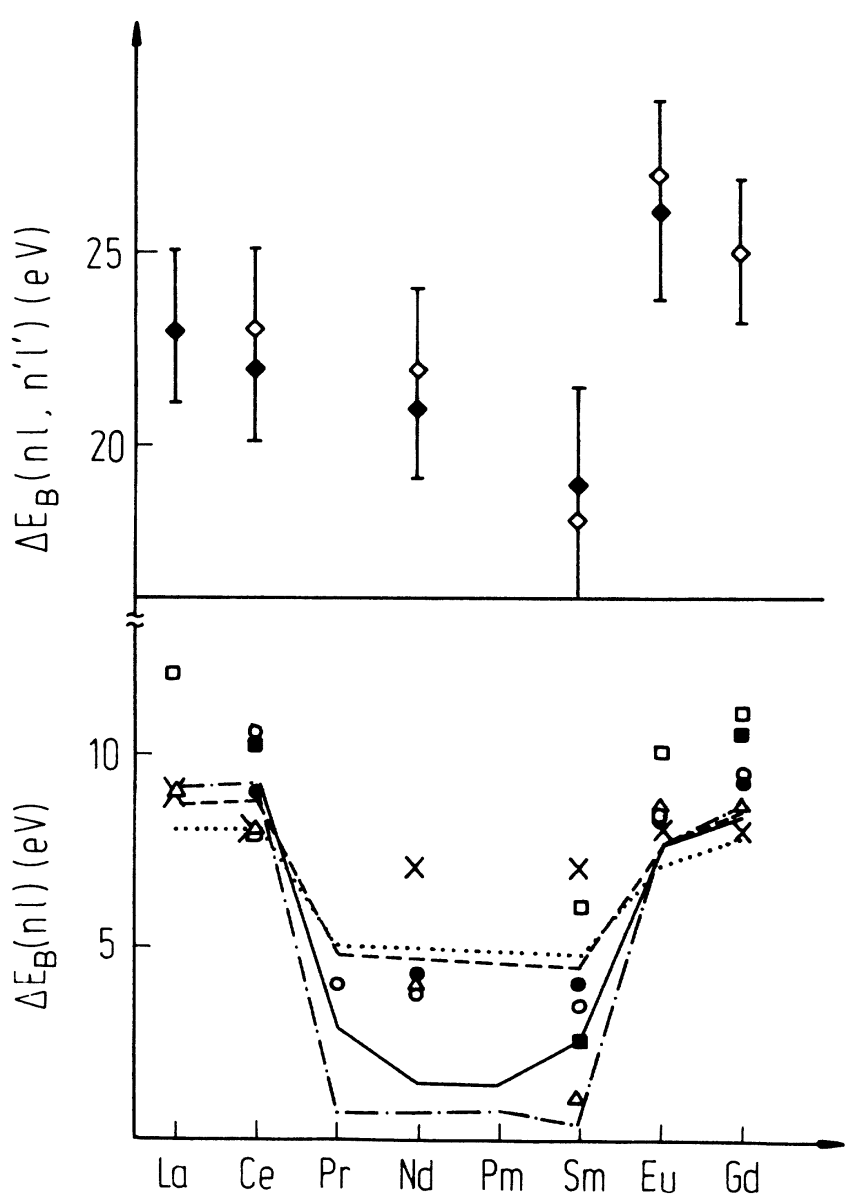

FIG. 1. Lower part: single-hole atom-to-metal bindingenergy shifts. Experimental values: $\square, \Delta E_{B}(2 p)$ from Ref. 25; $\triangle, \Delta E_{B}(4 d) ; \square, \Delta E_{B}(5 s) ; \times, \Delta E_{B}(5 p) ; \bullet$ and $\bigcirc, \Delta E_{B}(4 f) ; \bigcirc$ from Ref. 20. Calculated values (Ref. 20): -.-.-., $\Delta E_{B}(2 p)$; $-\Delta E_{B}(5 s) ; \cdots \cdot \Delta E_{B}(5 p) ;-, \Delta E_{B}(4 f)$. Upper part: Double-hole atom-to-solid binding-energy shift. $\nabla, \Delta E_{B}(5 p, 5 p)$; $\diamond, \Delta E_{B}(5 p, 4 f)$. 
been used as the reference energy levels for atomic and solid-state spectra, respectively.

\section{RESULTS AND DISCUSSION}

\section{Atom-to-metal binding-energy shifts $\Delta E_{B}(n l)$}

The photoelectron spectra of the rare earths which we have studied are often complicated by the strong coupling between the core hole created and the holes in the partially filled $4 f$ subshell. This coupling splits the photolines into several line components. In this study we have in most cases estimated the center of gravity of the overlapping components and then used these values for the binding energies of the corresponding subshell.

The atomic $E_{B}^{a}(n l)$ and the metallic $E_{B}^{m}(n l)$ bindingenergy values are given in Table $I$ and the experimental free-atom-to-metal binding-energy shifts $\Delta E_{B}(n l)$ $=E_{B}^{a}(n l)-E_{B}^{m}(n l)$ are plotted in Fig. 1. For the solidstate binding energies the values are taken from the collection given by Riviere et $a l .{ }^{23}$ Also included are the $2 p$ binding-energy-shift values for $\mathrm{Ce}, \mathrm{Sm}$, and $\mathrm{Gd}$ determined by Materlik et al. ${ }^{25}$ from absorption measure- ments and estimates ${ }^{20}$ for $4 f$ shifts based on optical freeatom data. The uncertainties of the atom-to-metal binding-energy shifts are given in Table I. For comparison, theoretical shift values calculated by Herbst ${ }^{20}$ are also included in Fig. 1. Inspection of Fig. 1 shows that the shifts of $2 p, 4 d$, and $4 f$ levels lie close together, with the $4 d$ shifts systematically slightly smaller and in good agreement with the calculated values. Only in the case of $\mathrm{Nd}$ are the experimental $4 f$ shifts approximately $2.5 \mathrm{eV}$ higher than the theoretical values. The $4 d$ and $4 f$ shifts also clearly follow the steplike changes between $\mathrm{Ce}$ and $\mathrm{Pr}$ and $\mathrm{Sm}$ and Eu when the configuration changes between the free atoms and solid metals start and end, respectively. Also, the $2 p$ binding-energy shifts of Materlik et al. ${ }^{25}$ for $\mathrm{Ce}, \mathrm{Sm}$, and $\mathrm{Gd}$ are very close to our $4 f$ binding-energy shifts for these systems. The experimentally observed close agreement between the $2 p, 4 d$, and $4 f$ shifts indicate that also $4 f$ levels are well-localized core levels in the metallic state.

The $5 p$ binding-energy shifts obtained for $\mathrm{Nd}$ and $\mathrm{Sm}$ and the $5 s$ shifts for Sm are essentially higher than their $4 d$ and $4 f$ shifts. In fact, all the $5 p$ shifts are in the range 7-9 $\mathrm{eV}$ and do not show as pronounced changes indicated by the theoretical values in going from $\mathrm{Ce}$ to $\mathrm{Pr}$ and from $\mathrm{Sm}$ to $\mathrm{Eu}$.

TABLE I. Binding energies $E_{B}(n l)$ of atomic and metallic (Ref. 23) La, Ce, Nd, Sm, Eu, and Gd. The atom-to-metal bindingenergy shifts $\Delta E_{B}(n l)$ are presented in separate columns. The $\Delta E_{B}(2 p)$ values have been taken from Ref. 25.

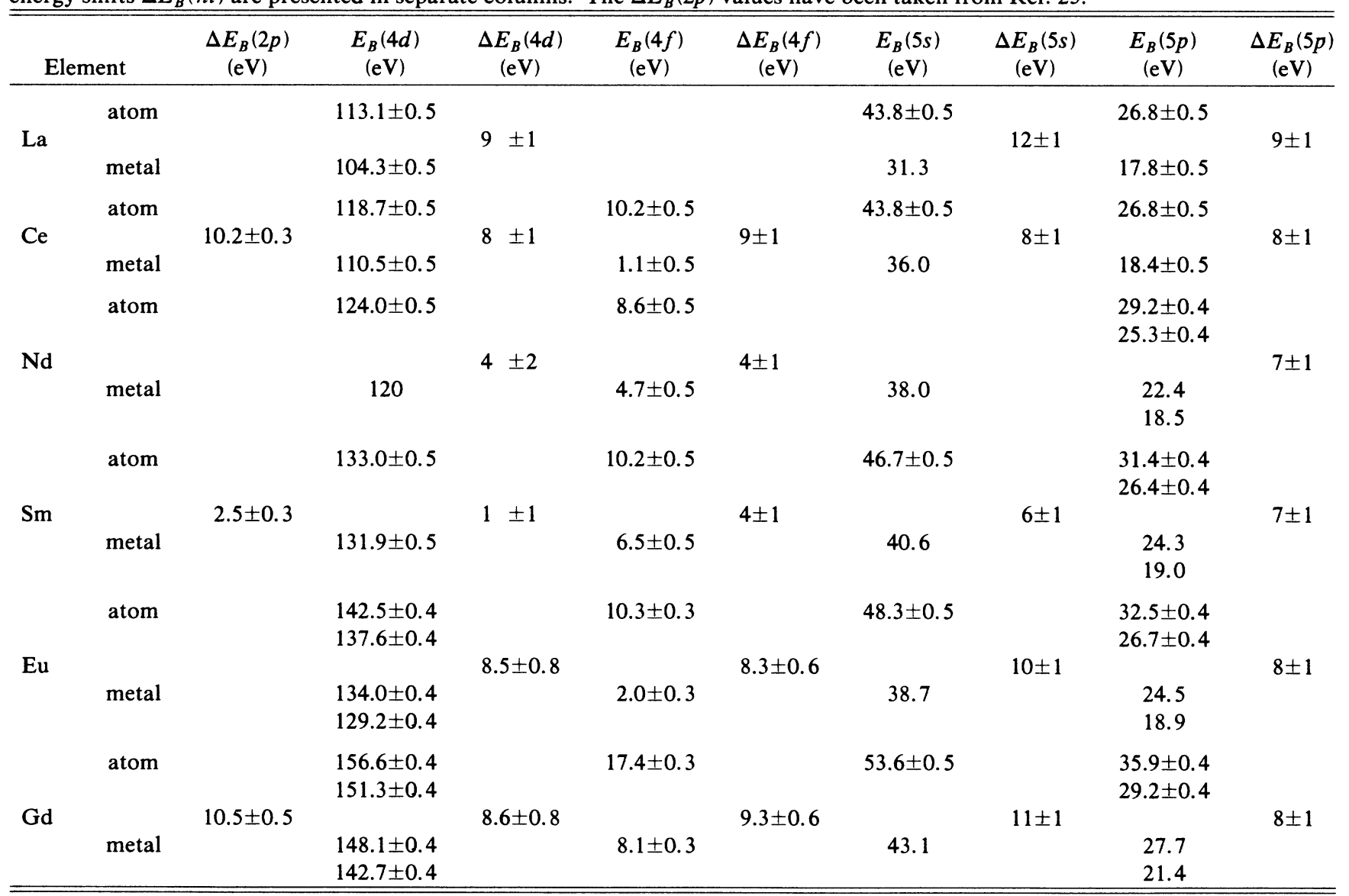




\section{Auger and recombination spectra}

The experimental vapor- and solid-phase electron emission spectra excited by white synchrotron radiation (SR) or electron impact $\left(e^{-}\right)$for $\mathrm{La}, \mathrm{Ce}, \mathrm{Pr}, \mathrm{Nd}, \mathrm{Sm}, \mathrm{Eu}, \mathrm{Gd}$, $\mathrm{Tb}, \mathrm{Dy}$, and $\mathrm{Tm}$ are shown in Figs. 2-6. A noticeable feature is the overall similarity between the electronexcited solid and vapor-phase spectra. Most of the spectra appear to consist of two dominating line groups, a lower-energy broad doublet followed by one or two broad structures at higher kinetic energies. In particular, the low-energy groups of the corresponding vapor and metal spectra show very similar structures. In Figs. 2-6, in order to make the visual comparison easier, the energy scales of the spectra are shifted so that the positions of the strongest low-energy peaks coincide. As in the case of unresolved transition arrays observed in the laserproduced plasma emission of rare-earth metals (see, e.g., O'Sullivan ${ }^{36}$ ), many Auger and autoionization transitions, respectively, blend to form the broad peaks dominating our spectra. The second characteristic feature of the spectra is that, although they show basically similar structures for both metal and atom, the vapor spectra

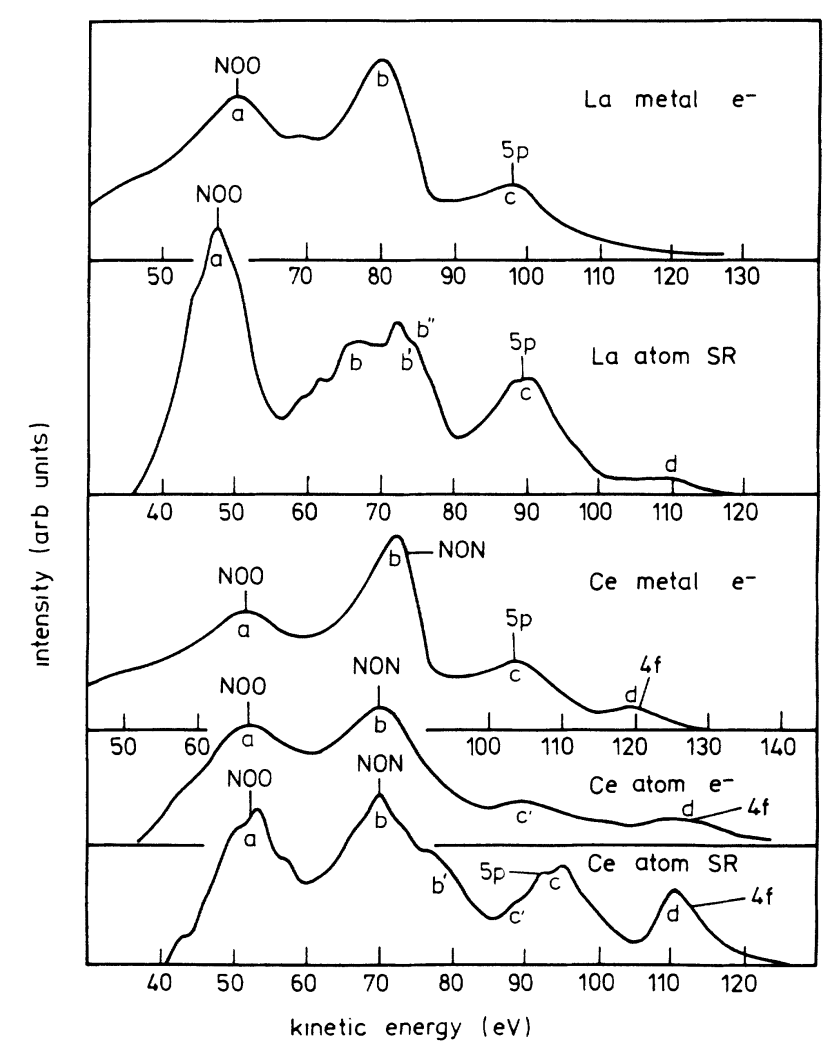

FIG. 2. Upper part: electron-excited Auger spectrum of metallic La (Ref. 23). Auger spectrum of atomic La excited by undispersed synchrotron radiation (SR). Lower part: electronexcited Auger spectra of metallic (Ref. 23) and atomic Ce. The spectrum excited by $\mathrm{SR}$ is presented by the bottom curve. The $4 d-4 f(4 f), 4 d-5 p(5 p)$ direct recombination lines and the $N_{4,5} O_{2,3} O_{2,3}(\mathrm{NOO}), \mathrm{N}_{4,5} \mathrm{O}_{2,3} N_{6,7}(\mathrm{NON})$ Auger lines are indicated. clearly extend further into the high-energy region, indicating that the highest-energy peaks originate from different transitions with much smaller metal-to-atom shifts than those of the main low-energy peaks. The spectra excited by high-energy electrons or white light arise from three different types of processes: $4 d$ Auger, $4 p$ Auger emission, and direct recombination emission. As pointed out by Riviere et al. ${ }^{23}$ the ionization probability for $4 p$ levels is about $26 \%$ of the ionization probability of $4 d$ levels by $2-\mathrm{keV}$ electrons. Using white-light excitation $4 d$ ionization can be expected to be still more dominating due to the higher cross section of $4 d$ levels and the fact that the large relative portion of the photon flux between $4 d$ and $4 p$ ionization thresholds contributes only to $4 d$ based spectra. Therefore the $4 d$ spectra can be expected to dominate, although some weaker spectral structures can originate from ionizaion of $4 p$ levels. Our identification of the main peaks agrees with that of Riviere $e t a l .{ }^{23}$ and is indicated in Figs. 2-6. The metal-toatom kinetic-energy shifts of the corresponding Auger lines, $\Delta E_{A}=E_{A}^{m}-E_{A}^{a}$, and of the direct recombination lines, $\Delta E_{\mathrm{DR}}=E_{\mathrm{DR}}^{m}-E_{\mathrm{DR}}^{a}$, can be read off the figures. Superscript $m$ stands for metal while superscript $a$ means an isolated atom is considered. The spectra shown in Figs. 2-6 will be briefly discussed separately.

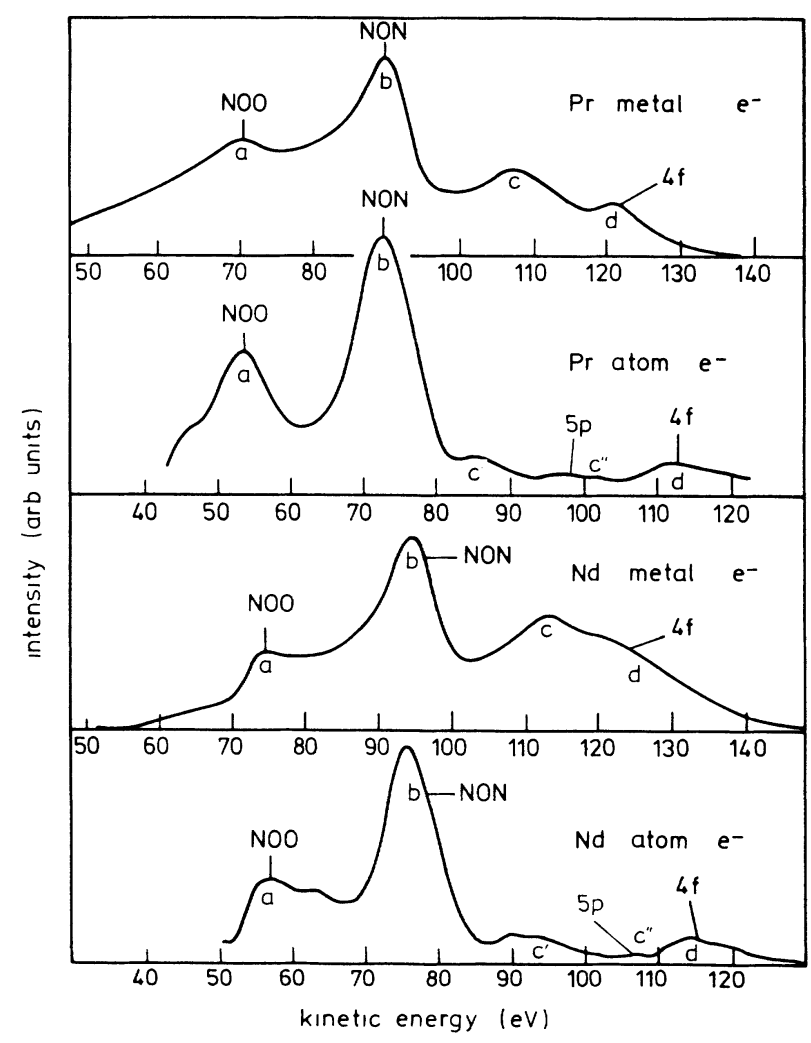

FIG. 3. Upper part: electron-excited Auger spectra of metallic (Ref. 23) and atomic Pr. Lower part: electron-excited Auger spectra of $\mathrm{Nd}$ metal (Ref. 23) and $\mathrm{Nd}$ atoms. The $4 d-4 f$ (4f), $4 d-5 p(5 p)$ direct recombination lines and the $N_{4,5} O_{2,3} O_{2,3}$ (NOO), $N_{4,5} \mathrm{O}_{2,3} N_{6,7}(\mathrm{NON})$ Auger lines are indicated. 


\section{$L a\left\{[X e] 5 d 6 s^{2}\right.$ atom, $\left[X e(5 d, 6 s)^{3}\right.$ metal $\left.]\right\}$}

The upper part of Fig. 2 shows the solid-state spectrum of La excited by $e^{-}$and that of the vapor phase excited by synchrotron radiation (SR). La has no $4 f$ electrons in the ground state, by outermost electrons being $5 d$ and $6 s$ which form the valence band in the metallic state. The peaks denoted by $a$ and $b$ can be identified as $\mathrm{N}_{4,5} \mathrm{O}_{2,3} \mathrm{O}_{2,3}$ and $\mathrm{N}_{4,5} \mathrm{O}_{2,3} \mathrm{~V}$ Auger transitions, respectively, and they show a metal-to-atom kinetic-energy shift of $14 \pm 1 \mathrm{eV}$. The structures $b^{\prime}$ and $b^{\prime \prime}$ do not have clear counterparts in the solid-state spectrum, but they are both shifted with respect to peak $b$ by approximately the same amount as the structure $c(7.5 \pm 1.0 \mathrm{eV})$; the latter is identified as the $4 d-5 p$ direct recombination (DR) line. The energy separation between $b^{\prime}, b^{\prime \prime}$, and $c$ corresponds to the $5 s-5 p$ binding-energy difference of $17 \mathrm{eV}$. Thus, the structures $b^{\prime}$ and $b^{\prime \prime}$ are most probably the $4 d-5 s$ recombination lines. The weak peak $d$ in the vapor spectrum, being approximately $20 \mathrm{eV}$ from peak $c$ in agreement with the $5 p$-to- $(5 d, 6 s)$ binding-energy difference, could be the $4 d-(5 d, 6 s)$ recombination line.

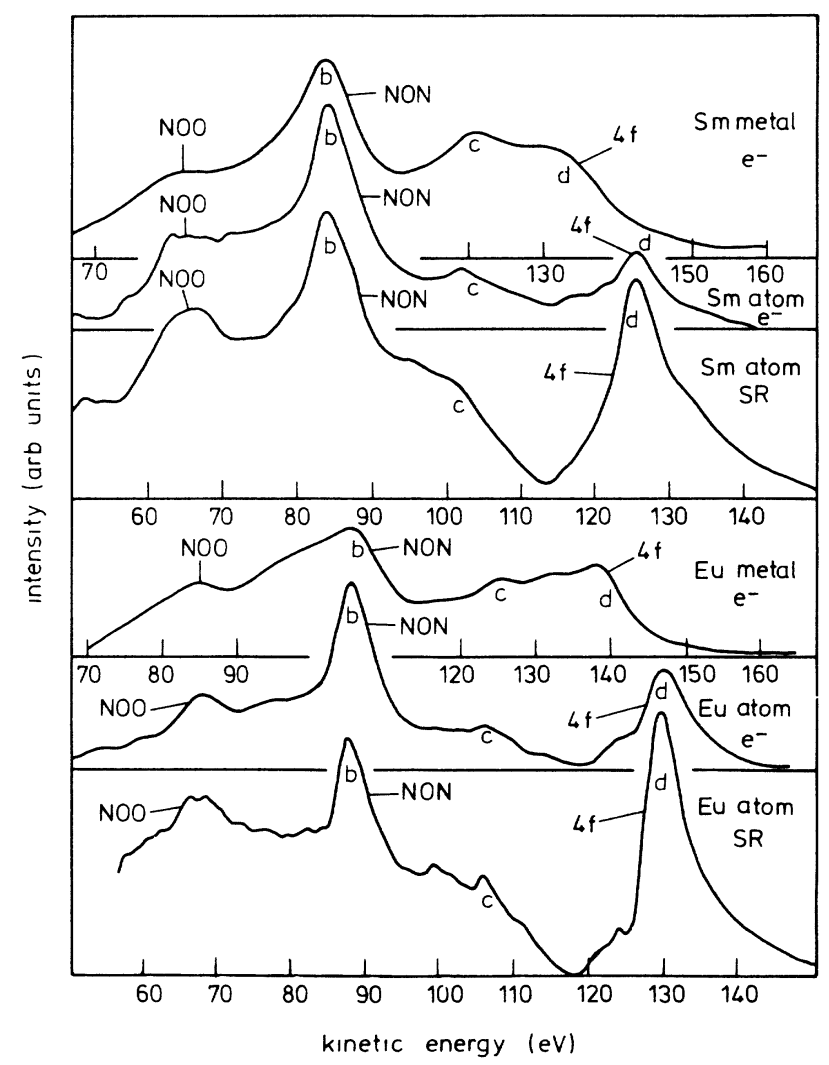

FIG. 4. Upper part: electron-excited Auger spectrum of metallic Sm (Ref. 23). Electron- and SR-excited Auger spectra of $\mathrm{Sm}$ atoms. Lower part: electron-excited Auger spectrum of metallic Eu (Ref. 23). Electron- and SR-excited Auger spectra of atomic Eu. The $4 d-4 f(4 f)$ direct recombination lines and the $\mathrm{N}_{4,5} \mathrm{O}_{2,3} \mathrm{O}_{2,3}$ (NOO), $\mathrm{N}_{4,5} \mathrm{O}_{2,3} \mathrm{~N}_{6,7}$ (NON) Auger lines are indicated.

$$
\text { Ce }\left[\ldots 4 f 5 d 6 s^{2}, 4 f(5 d, 6 s)^{3}\right]
$$

The $e^{-}$solid- and vapor-phase spectra of Ce shown in the lower part of Fig. 2 are similar. However, the SR light-excited atomic spectrum does reveal more structures and enhanced intensities in the high-energy region. Ce has one $4 f$ electron which also makes $4 d-4 f$ DR possible, creating peak $d$, which is strongly enhanced by SR. We obtain a value of $9 \pm 1 \mathrm{eV}$ for the $d-d, c-c$, and $b-b^{\prime}$ shifts, which supports the interpretation of $c$ to $4 d-5 p$ and $b^{\prime}$ to $4 d-5 s$ recombination lines. The shifts of structures $a, b$, and $c^{\prime}$ are $14-15 \mathrm{eV}$, as would be expected for Auger transitions.

$$
\operatorname{Pr}\left[\ldots 4 f^{3} 6 s^{2}, 4 f^{2}(5 d, 6 s)^{3}\right]
$$

$\operatorname{Pr}$ is the first element in this series which undergoes a change in its $4 f$ electron number going from the atomic to metallic ground state. The $e^{-}$spectra of metallic and atomic $\operatorname{Pr}$ (Fig. 3) deviate considerably at higher kinetic energies. Peaks $a$ and $b$, Auger transitions, have shifted by $16-17 \mathrm{eV}$ to higher energies in the metal. Peak $d$ is

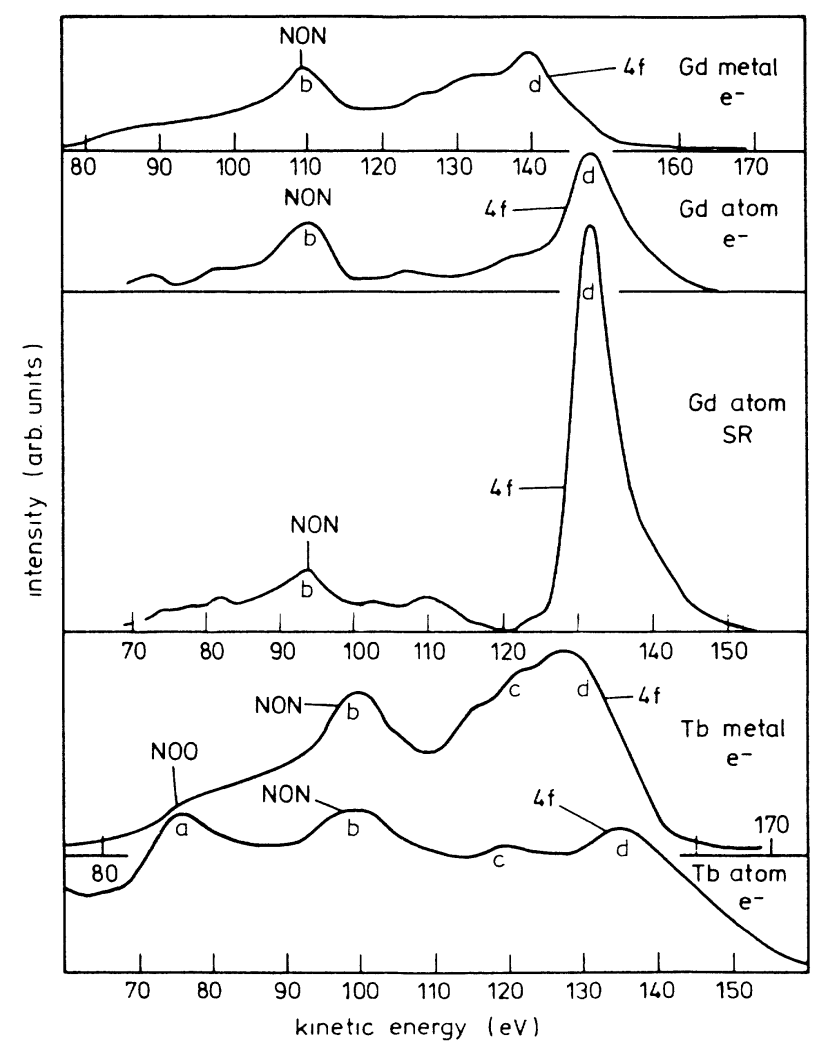

FIG. 5. Upper part: electron-excited Auger spectra of metallic (Ref. 23) and atomic Gd and SR-excited Auger spectrum of atomic Gd. Lower part: electron-excited Auger spectra of $\mathrm{Tb}$ metal (Ref. 23) and Tb atoms. The $4 d-4 f(4 f)$ direct recombination lines and the $\mathrm{N}_{4,5} \mathrm{O}_{2,3} \mathrm{O}_{2,3}$ (NOO), $\mathrm{N}_{4,5} \mathrm{O}_{2,3} \mathrm{~N}_{6,7}(\mathrm{NON})$ Auger lines are indicated. 
again the $4 d-4 f$ DR line with the shift of $8 \pm 1 \mathrm{eV}$. The weak structure $c^{\prime \prime}$ in the atom spectrum shows the same average shift with respect to $c$ in the metal spectrum. Thus it is most probably caused by $4 d-5 p$ DR. Peak $c$ in the metal is the superposition of the $4 d-5 p$ DR line and the Auger transitions; in the vapor spectrum peak $c^{\prime}$ is most probably the Auger transition $N_{4,5} N_{6,7} N_{6,7}$.

$$
N d\left[\ldots 4 f^{4} 6 s^{2}, 4 f^{3}(5 d, 6 s)^{3}\right]
$$

The spectra of $\mathrm{Nd}$ (Fig. 3) are rather similar to those of Pr, except that in the metal spectrum structures $c$ and $d$ are now more overlapping. It is obvious that $d$ arises from the $4 d-4 f \mathrm{DR}$, but its location in the metal spectrum is rather uncertain, the shift being estimated to be $7 \pm 2 \mathrm{eV}$. From peaks $a$ and $b$ we obtain an Auger shift of $17-18 \mathrm{eV}$. The structure $c$ in the metal spectrum probably is again a superposition of $N_{4,5} N_{6,7} N_{6,7}$ Auger and $4 d-5 p$ recombination lines, which are resolved in the atom spectrum $\left(c^{\prime}, c^{\prime \prime}\right)$.

$$
\operatorname{Sm}\left[\ldots 4 f^{6} 6 s^{2}, 4 f^{5}(5 d, 6 s)^{3}\right]
$$

The spectra of Sm shown in Fig. 4 display new and interesting features. The $e^{-}$vapor spectrum shows an in-

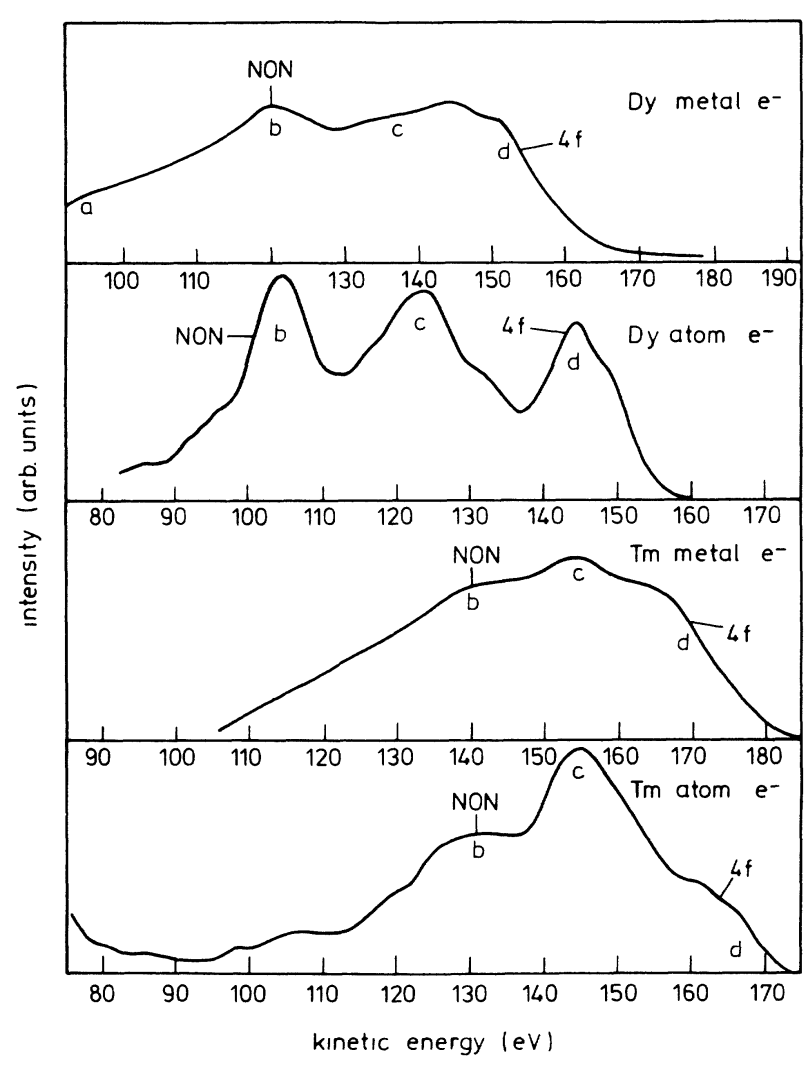

FIG. 6. Upper part: electron-excited Auger spectrum of metallic (Ref. 23) and atomic Dy. Lower part: electron-excited Auger spectra of metallic (Ref. 23) and atomic Tm. The $4 d-4 f$ $(4 f)$ direct recombination lines and the $N_{4,5} O_{2,3} N_{6,7}(N O N)$ Auger lines are indicated. tense $4 d-4 f$ DR peak, which is very strongly enhanced by the use of the SR. The lower-energy regions of the spectra are again almost identical. $N_{4,5} \mathrm{O}_{2,3} N_{6,7}$ Auger transitions form the most intense peak, $b$, and the intensity of the $4 d-4 f$ DR lines is clearly increasing as the number of $4 f$ electrons increases, making its energy position in the solid-state spectrum easier to estimate. Thus for the highest-energy peaks, $d$, we obtain a shift of $6 \pm 2 \mathrm{eV}$ and for the strongest $N_{4,5} O_{2,3} N_{6,7}$ peaks, $b$, a shift of $17 \pm 1$ $\mathrm{eV}$, the smaller shift confirming the autoionization origin of peaks $d$.

The origin of peak $c$ in the vapor spectrum requires closer consideration. Energetically it could be direct recombination line with a $5 p$-electron emission, or the normal $N_{4,5} N_{6,7} N_{6,7}$ Auger process. If it is the former, it should shift upon going from atom to metal by approximately the same amount as the $4 f$ recombination line, putting it at the bottom of the valley on the high-energy side of the strong peak $b$. Instead, peak $c$ in the solidstate spectrum has $19 \mathrm{eV}$ higher kinetic energy than in the atom spectrum, in reasonable agreement with the shift of the main peak. It can be concluded therefore that the peak $c$ at $102.5 \mathrm{eV}$ is a superposition of $N_{4,5} N_{6,7} N_{6,7}$ Auger and $4 d-5 p$ DR lines with a dominant Auger contribution.

$$
E u\left[\ldots 4 f^{7} 6 s^{2}, 4 f^{7}(5 d, 6 s)^{2}\right]
$$

Atomic Eu has a half-filled $4 f$ subshell which manifests itself as the sharpest peak structures [6-8 eV full width at half maximum (FWHM)] in the atomic spectrum. For the solid-state shifts we obtain $7 \pm 2$ and $18 \pm 1 \mathrm{eV}$ for the high-energy recombination peak $d$ and the strongest Auger peaks $b$, respectively. Following the line of the previous discussion for $\mathrm{Sm}$, the main origin of peak $c$ in the vapor spectrum would seem to be the superposition of Auger and DR-type transitions; peak $c$ again shows a slightly higher shift than the main Auger peak. Characteristic of the Eu solid-state spectrum is the apparent asymmetry of peak $b$.

\section{$G d\left[\ldots 4 f^{7} 5 d 6 s^{2}, 4 f^{7}(5 d 6 s)^{3}\right]$}

Gd also has a half-filled $4 f$ subshell. Taking this close similarity of electron configurations with $\mathrm{Eu}$ into account, the observed spectral change between them is surprising. The $4 d-4 f$ DR peak $d$ is the most intense in both phases. The solid-state shift of peak $d$ is $9 \pm 1 \mathrm{eV}$ and that of the $N_{4,5} O_{2,3} N_{6,7}$ Auger structure $b, 16 \pm 1 \mathrm{eV}$. The peak $d$ in the $e^{-}$solid-state spectrum of Gd was first suggested by Dufour and Bonnelle ${ }^{1}$ to originate from the $4 d-4 f$ DR process. The atom spectrum taken with $\mathrm{SR}$ shows the relative intensity of this $\mathrm{DR}$ peak to be greatly enhanced. The width (FWHM) of the DR line in the vapor spectrum is approximately $11 \mathrm{eV}$, with an electron spectrometer resolution of $1.0 \mathrm{eV}$.

The vapor-phase spectrum shows structure in the expected energy region of $\mathrm{N}_{4,5} \mathrm{O}_{2,3} \mathrm{O}_{2,3}$ Auger transitions $(70-85 \mathrm{eV})$, but this structure is almost completely smeared out in the solid-state spectrum. 


$$
\operatorname{Tb}\left[\ldots 4 f^{9} 6 s^{2}, 4 f^{8}(5 d 6 s)^{3}\right]
$$

In the spectra of $\mathrm{Tb}$ (Fig. 5) $4 d-4 f$ recombination lines $d$ in the metal spectrum appear as much broader features than in the case of $\mathrm{Gd}$, overlapping partly with $N_{4,5} N_{6,7}\left(N_{6,7}\right)$ Auger transitions $c$ and resulting in a large uncertainty on the value of the shift, of $8 \pm 2 \mathrm{eV}$. $N_{4,5} O_{2,3} N_{6,7}$ transitions $b$ form the best resolved "peaks," and are shifted by $16 \pm 2 \mathrm{eV}$. It is interesting to see how clearly distinguishable peak $a$ of $N_{4,5} \mathrm{O}_{2,3}, \mathrm{O}_{2,3}$ transitions becomes in vapor phase, whereas it can be barely located in the solid-state spectrum.

$$
\text { Dy }\left[\ldots 4 f^{10} 6 s^{2}, 4 f^{9}(5 d 6 s)^{3}\right]
$$

For Dy (Fig. 6) peak $a\left(N_{4,5} \mathrm{O}_{2,3} \mathrm{O}_{2,3}\right)$ has disappeared completely from the metal and vapor spectra, but peaks $b, c$, and $d$ are enhanced. The $4 d-4 f$ DR peak $d$ is particularly strong in the $e^{-}$vapor spectrum, but overlaps strongly with the Auger peaks in the solid-state spectrum. Our estimates for the shifts are $16 \pm 2,17 \pm 2$, and $7 \pm 2 \mathrm{eV}$ for peaks $b, c$, and $d$, respectively.

$$
\operatorname{Tm}\left[\ldots 4 f^{13} 6 s^{2}, 4 f^{12}(5 d 6 s)^{3}\right]
$$

In going to Tm (Fig. 6) the relative intensity of the $4 d$ $4 f$ DR $(d)$ decreases markedly, the structures for the solid-state spectrum being almost completely smeared out. The observed shifts between the structures $b, c$, and $d$ are surprisingly small, $10 \pm 3,10 \pm 2$, and $7 \pm 3 \mathrm{eV}$, respectively.

\section{Metal-to-atom shifts of DR lines}

The experimental values for the metal-to-atom shifts $\Delta E_{\mathrm{DR}}$ of the $4 d-4 f$ recombinaion lines studied are shown in Fig. 7. The recombination process creates a single hole state analogously with photoemission. Hence the metalto-atom shifts, caused mainly by extra-atomic relaxation of surrounding charge distribution in the metals, could be of about the same magnitude. In fact, our shift values typically span the range $6-9 \mathrm{eV}$, and we obtain similar values for binding-energy shifts. However, the $4 d-4 f$ DR and $4 f$ binding-energy shifts are different. For example, DR shifts do not display the same changes as bindingenergy shifts for those elements which undergo valence change between atomic and metallic states.

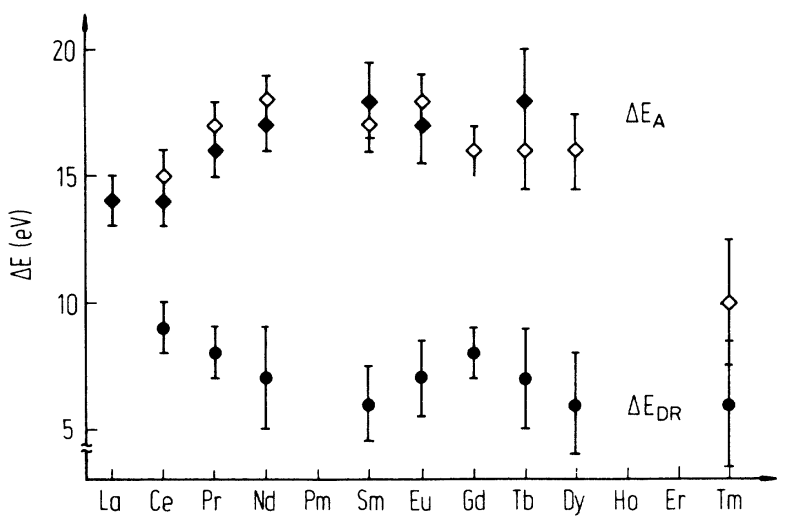

FIG. 7. Metal-to-atom kinetic-energy shifts of the Auger, $\Delta E_{A}$, and the direct recombination lines, $\Delta E_{\mathrm{DR}}$

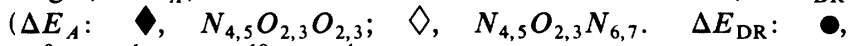
$\left.4 d^{9} 4 f^{m+1} n l \rightarrow 4 d^{10} 4 f^{m-1} \epsilon l^{\prime}\right)$.

The direct recombination of a $4 d$ hole could be described energetically as a two-step process, where one $4 d$ electron is first excited to a $(4 f, \epsilon f)$ resonance state and then decays with the emission of, for example, one $4 f$ electron. The kinetic energy of the emitted electron is then given by the difference of $4 d \rightarrow(4 f, \epsilon f)$ resonance excitation energy $E_{4 d \rightarrow 4 f, \epsilon f}$ and $4 f$ binding energy, where the same energy reference level is used for the binding and kinetic energies. The solid-state shift is thus given by

$$
\Delta E_{\mathrm{DR}}=\Delta E_{4 d \rightarrow 4 f, \epsilon f}+\Delta E_{B}(4 f),
$$

with

$$
\Delta E_{4 d \rightarrow 4 f, \epsilon f}=E_{4 d \rightarrow 4 f, \epsilon f}^{m}-E_{4 d \rightarrow 4 f, \epsilon f}^{a} .
$$

Experimental values for $4 d \rightarrow(4 f, \epsilon f)$ giant resonance excitation energies can be obtained from the measurements of partial cross section $\sigma(4 f)$ for atomic and solid lanthanides. ${ }^{11}$ These data for $\mathrm{Ce}, \mathrm{Nd}, \mathrm{Sm}, \mathrm{Eu}$, and $\mathrm{Gd}$ are given in Table II, which also includes the present $4 f$ binding-energy shifts and, calculated from them, $4 d-4 f$ DR shifts, together with the experimental values. It can be seen that the agreement between the "calculated" and experimental DR shifts is excellent within the limits of the experimental uncertainties.

TABLE II. Energy of the $4 d \rightarrow(4 f, \epsilon f)$ resonance, $E_{4 d \rightarrow 4 f, \epsilon f}$, for metallic (Ref. 23) and atomic Ce, $\mathrm{Nd}, \mathrm{Sm}, \mathrm{Eu}$, and $\mathrm{Gd}$. The shift of this resonance energy, $\Delta E_{4 d \rightarrow 4 f, \epsilon f}$, and the atom-to-metal $4 f$ binding-energy shift $\Delta E_{B}(4 f)$ are given in columns 4 and 5 . Columns 6 and 7 show the metal-to-atom kinetic-energy shifts of the direct recombination lines, $\Delta E_{\mathrm{DR}}(4 f)$, obtained from the model discussed in the text and the experimental values.

\begin{tabular}{ccccccc}
\hline & \multicolumn{2}{c}{$\Delta E_{4 d \rightarrow 4 f, \epsilon f}(\mathrm{eV})$} & & \multicolumn{2}{c}{$\Delta E_{\mathrm{DR}}(4 f)(\mathrm{eV})$} \\
Element & Metal & Atom & $\Delta E_{4 d \rightarrow 4 f, \epsilon f}(\mathrm{eV})$ & $\Delta E_{B}(4 f)(\mathrm{eV})$ & Model & Experiment \\
\hline Ce & $121.3 \pm 0.3$ & $121.5 \pm 0.3$ & $-0.2 \pm 0.6$ & $9 \pm 1$ & $9 \pm 2$ & $9 \pm 1$ \\
Nd & $131.0 \pm 0.5$ & $128.0 \pm 0.5$ & $3 \pm 1$ & $4 \pm 1$ & $7 \pm 2$ & $7 \pm 2$ \\
Sm & $138.8 \pm 0.5$ & $134.8 \pm 0.3$ & $4.0 \pm 0.8$ & $4 \pm 1$ & $8 \pm 2$ & $6 \pm 2$ \\
Eu & $140.8 \pm 0.3$ & $140.7 \pm 0.3$ & $0.1 \pm 0.6$ & $8.3 \pm 0.6$ & $8 \pm 1$ & $7 \pm 2$ \\
Gd & $149.5 \pm 0.3$ & $149.6 \pm 0.3$ & $-0.1 \pm 0.6$ & $9.3 \pm 0.6$ & $9 \pm 1$ & $9 \pm 1$ \\
\hline \hline
\end{tabular}


From the values of Table II it can be seen that for $\mathrm{Nd}$ and $\mathrm{Sm}$, which change valence, $4 d \rightarrow(4 f, \epsilon f)$ excitation energy shifts are rather large (3-4 eV) compared to those of the other elements $(<1 \mathrm{eV})$. This almost completely compensates for the lower $4 f$ binding-energy shifts of the valence-changing elements and results in a smooth change in the DR shifts as a function of $Z$.

\section{Auger-energy shifts}

Experimental metal-to-atom kinetic-energy shifts of the Auger peaks, $\Delta E_{A}$, are also plotted in Fig. 7. The results show a smooth variation with atomic number $Z$ and, similar to the DR shifts, do not display prominent changes for the valence-changing elements analogously with the binding-energy shifts. However, between $\mathrm{Ce}$ and Pr there is a clear increase of about $2.5 \mathrm{eV}$ in the Auger shifts, but there is a significant change between $\mathrm{Sm}$ and Eu. The overall magnitude of the Auger shifts is very close to that found ${ }^{26}$ experimentally for $4 d$ elements Pd-Te, taking into account that the solid-state energies are now referred to the Fermi level. Another noticeable feature of the present results is that the shifts for different Auger transitions, $\mathrm{N}_{4,5} \mathrm{O}_{2,3} \mathrm{O}_{2,3}$ and $\mathrm{N}_{4,5} \mathrm{O}_{2,3} \mathrm{~N}_{6,7}$, seem to be almost the same. The Auger energy $E_{A}(i j k)$ is the energy difference between the single $E_{B}(i)$ and the double-hole binding energies $E_{B}(j k)$,

$$
E_{A}(i j k)=E_{B}(i)-E_{B}(j k) .
$$

As a consequence, the shift of the Auger energy is given by the difference of the double-hole atom-to-metal shift $\Delta E_{B}(j k)=E_{B}^{a}(j k)-E_{B}^{m}(j k)$ and the single-hole atom-tometal shift $\Delta E_{B}(i)$,

$$
\Delta E_{A}(i j k)=\Delta E_{B}(j k)-\Delta E_{B}(i) .
$$

The double-hole energy shift is the real new information contained in the Auger-energy shifts. The experimental results for $\Delta E_{A}\left(N_{4,5} O_{2,3} O_{2,3}\right), \Delta E_{A}\left(N_{4,5} O_{2,3} N_{6,7}\right)$, $\Delta E_{B}(5 p, 5 p)$, and $\Delta E_{B}(5 p, 4 f)$ are summarized in Table III. The double-hole atom-to-metal binding-energy shifts are also displayed in the upper part of Fig. 1. The values smoothly decrease from $\mathrm{La}$ to $\mathrm{Sm}$, but there is a dramatic increase between $\mathrm{Sm}$ and $\mathrm{Eu}$. The double-hole bindingenergy shifts of $\mathrm{Eu}$ and $\mathrm{Gd}$ are approximately $7 \mathrm{eV}$ higher than the values for $\mathrm{Sm}$. The ratios $\Delta E_{B}(5 p, 5 p) / \Delta E_{B}(5 p)$ and $\Delta E_{B}(5 p, 4 f) / \Delta E_{B}(5 p)$ are close to 3. The ratio $\Delta E_{B}(5 p, 4 f) / \Delta E_{B}(4 f)$ oscillates between 2 and 7 , reflecting the changes of the $4 f$ binding-energy shift. The absolute values for the double-hole bindingenergy shifts and the ratio of 3 are consistent with the data presented for a series of elements by Mårtensson et al. ${ }^{22}$ The high single- and double-hole atom-to-metal binding-energy shifts for $\mathrm{Eu}$ and $\mathrm{Gd}$ are due to the stability of the $4 f^{7}$ configuration in both phases. The $4 f \rightarrow 5 d$ valence change upon condensation of $\mathrm{Nd}$ and $\mathrm{Sm}$ results in a reduction of the double-hole binding-energy shifts. Different screening channels may add to the observed differences in the atom-to-metal binding-energy shifts. In this respect the importance of $4 f$ screening forms the most intriguing problem.

The basic problem in undertaking a detailed investigation of these Auger spectra and the metal-to-atom Auger shifts is that, due to very large multiplet splitting both in the "initial" single-core-hole states and especially in the double-hole final states, the intensities of Auger transitions are divided between an extremely high number of overlapping line components. This manifests itself as very broad structures in the experimental spectra, often making the location of the peak positions inaccurate. An extra complication arises from the change of the $4 f$ electron number for valence-changing systems, resulting in different multiplet structures in free atoms and metals. The broad structures frustrate any attempt to determine the configuration of the two-hole final states from the splitting of the Auger lines.

We will first consider the metal-to-atom Auger-energy shifts in a simple model with the aid of one electron binding energies. The Auger electron $E_{A}(i j k)$ of the $(i j k)$ transitions can be written, neglecting fine-structure term symbols, as

$$
E_{A}(i j k)=E_{B}(i)-E_{B}(j)-E_{B}(k)-F(j k)+R_{A},
$$

where $E_{B}$ 's are binding energies, $F(j k)$ is the interaction energy between the two final-state holes, and $R_{A}$ is the additional relaxation energy caused by the creation of the second final-state hole in the presence of the first. The metal-to-atom Auger-energy shift is thus

$$
\begin{aligned}
\Delta E_{A}(i j k)= & -\Delta E_{B}(i)+\Delta E_{B}(j)+\Delta E_{B}(k) \\
& -\Delta F(j k)+\Delta R_{A} .
\end{aligned}
$$

The most simplified model, which assumes that

TABLE III. Metal-to-atom kinetic-energy shifts, $\Delta E_{A}$, of the $N_{4,5} O_{2,3} O_{2,3}$ and $N_{4,5} O_{2,3} N_{6,7}$ Auger lines. The last two columns give the atom-to-metal double-hole binding-energy shifts $\Delta E_{B}\left(n l, n^{\prime} l^{\prime}\right)$.

\begin{tabular}{ccccc}
\hline \hline Element & $\begin{array}{c}\Delta E_{A}\left(N_{4,5} O_{2,3} O_{2,3}\right) \\
(\mathrm{eV})\end{array}$ & $\begin{array}{c}\Delta E_{A}\left(N_{4,5} O_{2,3} N_{6,7}\right) \\
(\mathrm{eV})\end{array}$ & $\begin{array}{c}\Delta E_{B}(5 p, 5 p) \\
(\mathrm{eV})\end{array}$ & $\begin{array}{c}\Delta E_{B}(5 p, 4 f) \\
(\mathrm{eV})\end{array}$ \\
\hline $\mathrm{La}$ & $14 \pm 1$ & & $23 \pm 2$ & \\
$\mathrm{Ce}$ & $14 \pm 1$ & $15 \pm 1$ & $22 \pm 2$ & $23 \pm 2$ \\
$\mathrm{Nd}$ & $17 \pm 1$ & $18 \pm 1$ & $21 \pm 2$ & $22 \pm 2$ \\
$\mathrm{Sm}$ & $18 \pm 2$ & $17 \pm 1$ & $19 \pm 3$ & $18 \pm 2$ \\
$\mathrm{Eu}$ & $17 \pm 2$ & $18 \pm 1$ & $26 \pm 2$ & $27 \pm 2$ \\
$\mathrm{Gd}$ & & $16 \pm 1$ & & $25 \pm 2$ \\
\hline \hline
\end{tabular}




$$
\begin{aligned}
& \Delta E_{B}(i)=\Delta E_{B}(j)=\Delta E_{B}(k), \\
& \Delta F(j k)=0, \quad \Delta R_{A}=\text { const },
\end{aligned}
$$

predicts that the Auger shifts should show the same lower shift values for valence-changing systems as the binding energies. The experimental values, however, show just the opposite trend between $\mathrm{Ce}$ and $\mathrm{Pr}$ and no significant change between $\mathrm{Sm}$ and $\mathrm{Eu}$. The obvious oversimpifications of this approximation are assumptions of the equal binding-energy shifts for different $(n l)$ levels and that the hole-hole interaction energy remains unchanged for valence-changing systems in both phases. Our results for $5 p$ binding-energy shifts are significantly higher for $\mathrm{Nd}$ and $\mathrm{Sm}$ than the corresponding $4 d$ and $4 f$ shifts, which are the same within our experimental accuracy. For $\mathrm{Pr}, \mathrm{Nd}$, and $\mathrm{Sm}$ this should cause larger shifts for the $\mathrm{N}_{4,5} \mathrm{O}_{2,3} \mathrm{O}_{2,3}$ peaks than for the $\mathrm{N}_{4,5} \mathrm{O}_{2,3} \mathrm{~N}_{6,7}$ peaks of the same elements. However, this is not confirmed by the experimental results, indicating that this simple consideration of the binding-energy shifts fails to explain the Auger shifts.

Possible changes of hole-hole interaction energy and additional relaxation energies can be studied by using the experimental binding and Auger energies to calculate the quantity

$$
U_{\mathrm{eff}}=F(j k)-R_{A} .
$$

Values of $U_{\text {eff }}$ are plotted in Fig. 8 for both vapor and solid-state results from $\mathrm{La}$ to $\mathrm{Gd}$. The uncertainties of the experimental binding energies and especially the Auger energies cause a large scatter in the data which combine these quantities. However, some trends can be seen. The atomic $U_{\text {eff }}$ values do not vary dramatically, but show rather a steady increase from La to Gd. Only the values for Sm lie approximately $3 \mathrm{eV}$ below the value obtained by simple interpolation. The metallic $U_{\text {eff }}$ values are almost constant. If the hole-hole interaction energy is supposed to remain unchanged in going from free atoms to metal, the differences in $U_{\text {eff }}$ are due to the extra-atomic relaxation energy of metal. Our data indicate a considerable increase of this relaxation energy from La to $\mathrm{Gd}$. The significantly higher relaxation for the hole combination including the $4 f$ orbital is consistent with the notion that the $4 f$ holes are much better localized than the more diffuse $5 p$ holes.

An alternative method of studying experimental Auger shifts could be based on the comparison with the estimates obtained from the thermochemical model exten-

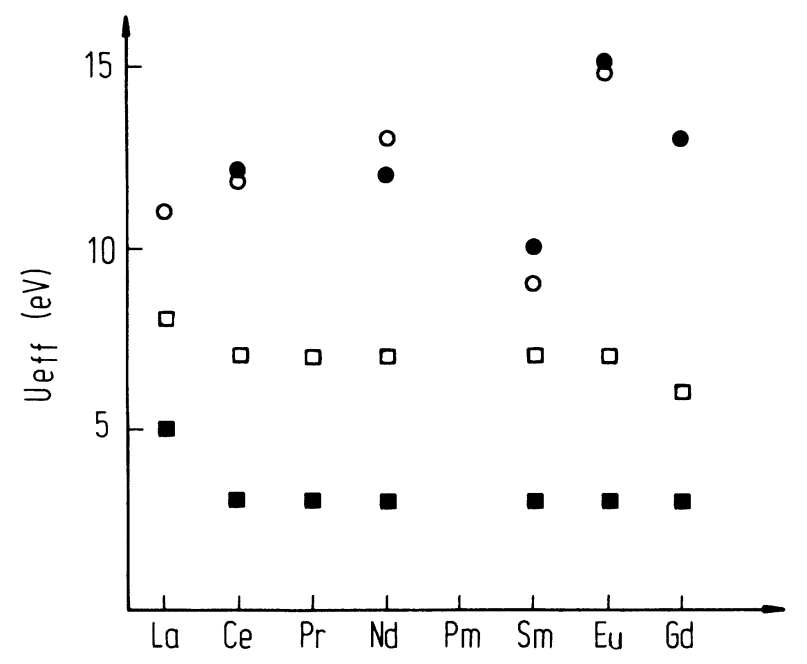

FIG. 8. $U_{\text {eff }}$ values for solid (Ref. 23) $\mathrm{N}_{4,5} \mathrm{O}_{2,3} \mathrm{O}_{2,3}$ ( $\square$ ), $N_{4,5} O_{2,3} N_{6,7}(V)(\square)$, atomic $N_{4,5} O_{2,3} O_{2,3}(\mathrm{O})$, and atomic $N_{4,5} O_{2,3} N_{6,7}(\bullet)$ transitions. The uncertainties of the atomic values are approximately $\pm 2.5 \mathrm{eV}$.

sively used by Johansson and Mårtensson, ${ }^{21,22}$ which has been found to give good estimates for binding- and Auger-energy solid-state shifts for several elements. Unfortunately, for most of the rare earths studied here the second ionization potentials, needed in this model in addition to the first ionization potentials and cohesive energy values, are not available or are very inaccurate. However, by applying the values given by Martin et $a l^{37}$ and published cohesive energies, ${ }^{38}$ we were able to obtain a rough estimate of $19 \pm 4 \mathrm{eV}$ for the metal-to-atom Auger shift of $\mathrm{Eu}$, in good agreement with our value of $18 \mathrm{eV}$ for $N_{4,5} O_{2,3} N_{6,7}$ transitions.

\section{ACKNOWLEDGMENTS}

The authors wish to thank the BESSY and HASYLAB staffs for continuous assistance. The financial support by the Bundesministerium für Forschung und Technologie der Bundesrepublik Deutschland and by the Deutsche Forschungsgemeinschaft is gratefully acknowledged. One of us (S.A.) is indebted to the Alexander-vonHumboldt Foundation, the Finnish Academy of Science, and Suomen Kulttuurirahasto for support making his participation in this work possible.
${ }^{1}$ G. Dufour and C. Bonnelle, J. Phys. (Paris) Lett. 35, L255 (1974).

${ }^{2}$ G. Dufour, R. C. Karnatak, J. M. Mariot, and C. Bonnelle, J. Phys. (Paris) Lett. 37, L119 (1976).

${ }^{3}$ F. P. Netzer, E. Bertel, and J. A. D. Matthew, J. Phys. C 14, 1891 (1981).

${ }^{4}$ J. A. D. Matthew, G. Strasser, and F. P. Netzer, J. Phys. C 15, L1019 (1982).

${ }^{5}$ F. Gerken, J. Barth, K. L. I. Kobayashi, and C. Kunz, Solid
State Commun. 35, 179 (1980).

${ }^{6}$ J. A. D. Matthew and S. M. Girvin, Phys. Rev. B 24, 2249 (1981).

${ }^{7}$ E. Schmidt, H. Schröder, B. Sonntag, H. Voss, and H. E. Wetzel, J. Phys. B 17, 707 (1984).

${ }^{8}$ T. M. Zimkina, V. A. Fomichev, S. A. Gribovski, and I. I. Zhukova, Fiz. Tverd. Tela (Leningrad) 9, 1447 (1967) [Sov. Phys. - Solid State 9, 1128 (1967)].

${ }^{9}$ R. Haensel, P. Rabe, and B. Sonntag, Solid State Commun. 8, 
1845 (1970).

${ }^{10}$ M. H. Hecht and I. Lindau, Phys. Rev. Lett. 47, 827 (1981).

${ }^{11} \mathrm{~F}$. Gerken, J. Barth, and C. Kunz, in X-Ray and Atomic Inner-Shell Physics, 1982, AIP Conf. Proc. No. 94, edited by B. Crasemann (AIP, New York, 1982), p. 602.

${ }^{12}$ P. Trebbia and C. Colliex, Phys. Status Solidi B 58, 523 (1973).

${ }^{13}$ M. W. D. Mansfield and J. P. Connerade, Proc. R. Soc. London, Ser. A 352, 125 (1976).

${ }^{14}$ H. W. Wolff, R. Bruhn, and B. Sonntag, Phys. Lett. 59A, 67 (1976).

${ }^{15}$ E. R. Radtke, J. Phys. B 12, L71 (1979).

${ }^{16}$ Th. Prescher, M. Richter, E. Schmidt, B. Sonntag, and H. E. Wetzel, J. Phys. B 19, 1645 (1986).

${ }^{17}$ M. Meyer, Th. Prescher, E. v. Raven, M. Richter, E. Schmidt, B. Sonntag, and H. E. Wetzel, Z. Phys. D 2, 347 (1986).

${ }^{18}$ U. Becker, H. G. Kerkhoff, D. W. Lindle, P. H. Kobriun, T. A. Ferret, P. A. Heimann, C. M. Truesdale, and D. A. Shirley, Phys. Rev. A 34, 2858 (1986).

${ }^{19}$ Giant Resonances in Atoms, Molecules and Solids, edited by J. P. Connerade, J. M. Esteva, and R. C. Karnatak (Plenum, New York, 1987).

${ }^{20}$ J. F. Herbst, Phys. Rev. B 28, 4204 (1983).

${ }^{21}$ B. Johansson and N. Mårtensson, Phys. Rev. B 21, 4427 (1980).

${ }^{22}$ N. Mårtensson, P. Hedegard, and B. Johansson, Phys. Scr. 29, 154 (1984).
23J. C. Riviere, F. P. Netzer, G. Rosina, and G. Strasser, J. Electron Spectrosc. Relat. Phenom. 36, 331 (1985).

${ }^{24} \mathrm{M}$. Richter, thesis, University of Hamburg (unpublished).

${ }^{25} \mathrm{G}$. Materlik, B. Sonntag, and M. Tausch, Phys. Rev. Lett. 51, 1300 (1983).

${ }^{26}$ D. A. Shirley, Phys. Rev. A 7, 1520 (1973).

${ }^{27}$ N. D. Lang and A. R. Williams, Phys. Rev. B 20, 1369 (1979).

${ }^{28}$ W. Kohn and L. J. Sham, Phys. Rev. 140, A1133 (1965).

${ }^{29}$ U. von Barth and L. Hedin, J. Phys. C 5, 1629 (1979).

${ }^{30}$ R. M. Nieminen and M. J. Puska, Phys. Rev. B 25, 67 (1982).

${ }^{31}$ S. Aksela, R. Kumpula, H. Aksela, J. Väyrynen, R. M. Nieminen, and M. Puska, Phys. Rev. B 23, 4362 (1981).

${ }^{32}$ S. Aksela, R. Kumpula, H. Aksela, J. Väyrynen, Phys. Scr. 25, 45 (1982).

${ }^{33}$ G. Hohlneicher, H. Pulm, and H. J. Freund, J. Electron Spectrosc. Relat. Phenom. 37, 209 (1985).

${ }^{34}$ N. J. Castellani and D. B. Leroy, J. Electron Specrosc. Relat. Phenom. 42, 161 (1987).

${ }^{35}$ T. Prescher, M. Richter, B. Sonntag, and H. E. Wetzel, Nucl. Instrum. Methods A 254, 627 (1987).

${ }^{36}$ G. O'Sullivan, in Giant Resonances in Atoms, Molecules and Solids, Ref. 19, p. 505.

${ }^{37}$ W. C. Martin, R. Zalubas, and L. Hagen, Atomic Energy Levels, The Rare-Earth Elements, Natl. Bur. Stand. (U.S.) Spec. Publ. No. 60, (U.S. GPO, Washington, D.C., 1978).

${ }^{38}$ B. Johansson, Phys. Rev. B 20, 1315 (1979). 